\title{
Empathy concerns enhance prosociality and modulate frontal theta oscillatory activity in young adults
}

Claudio Lavín ( $\square$ claudio.lavin@gmail.com )

Pontificia Universidad Católica de Chile

\section{Patricia Soto-Icaza}

Universidad del Desarrollo

\section{Vladimir López}

Pontificia Universidad Católica de Chile

\section{Pablo Billeke}

Universidad del Desarrollo

\section{Research Article}

Keywords: Decision-making, prosociality, empathy

Posted Date: November 23rd, 2021

DOI: https://doi.org/10.21203/rs.3.rs-1093058/v1

License: (c) (i) This work is licensed under a Creative Commons Attribution 4.0 International License. Read Full License 


\section{Abstract}

Decision making is a process that can be strongly affected by social factors. Profuse evidence has shown how people deviate from traditional rational-choice predictions under different levels of social interactions. The emergence of prosocial decision making, defined as any action that is addressed to benefit another individual even at the expense of personal benefits, has been reported as an important example of such social influence. Furthermore, brain evidence has shown the involvement of structures such the prefrontal cortex, anterior insula and midcingulate cortex during decision settings in which a decision maker interacts with others under physical pain or distress or while being observed by others. Using a slightly modified version of the dictator game, we tested the hypothesis that the inclusion of another person into the decision setting increases prosocial decisions in young adults and that this increase is higher when the other person is associated with others in need. At the brain level, we hypothesized that the increase in prosocial decisions correlates with frontal theta activity as a marker of empathy saliency. The results showed that the inclusion of another person into the decision setting increased prosocial behavior only when this presence was associated with someone in need and that this was associated with an increase in frontocentral theta-oscillatory activity. These results suggest that the presence of someone in need enhances both empathy concerns and norm compliance, raising the participants' prosocial decision making.

\section{Introduction}

In everyday life, there are several examples of decisions that could have direct consequences on others, such as deciding how much money to donate to charity, how much food to buy when there are supply problems or whether to give back a wallet full of cash that has just fallen down from someone's pocket. In all these cases, people may take into account, to different extents, the consequences that their own behavior has on the wellbeing of both others and themselves. This processing is crucially affected by the circumstances in which the decision has to be made. For example, to be observed by others or to observe someone who is under physical pain or distress changes our tendency to take into account others' wellbeing in our decisions. Although the influence of others seems clear, the psychological and neurobiological mechanisms underlying this process remain unclear.

Prosocial behavior refers to any action that is addressed to benefit another individual ${ }^{1}$, and in its extreme form of altruism, it implies a costly act that confers benefits to others ${ }^{2}$. Although traditional approaches assume a primarily selfish impulse of the maximization of immediate utilities in every human decision ${ }^{3}$, several empirical data have shown a much more complex scenario in which social and emotional factors, together with concerns about their own and others' wellbeing, explain people's social preferences ${ }^{1,2}$. Indeed, consistent evidence has shown that prosocial behavior can be associated not only with direct and indirect benefits to the decision-maker but also with his/her concerns about others' well-being. In this line, empathy, which has been defined as the sharing and comprehension of others' feelings and thoughts ${ }^{4}$, is 
an important modulator of prosociality by enhancing the presence of the other within the decision setting 4

There is evidence showing the impact that the presence of others has on subjects' decisions. The way that other persons appear varies depending on the experimental settings and goes from eyespots on the wall observing people's decisions to helping others under "real" physical pain. Evidence comparing public and anonymous decisions has described that individuals behave in a more prosocial way and adjust their behavior to a norm while they are being observed ${ }^{5-14}$. This evidence has suggested that, through emotional resonance and perspective-taking, the representation of another person can influence both decisions and brain activity associated with that process. Accordingly, different experimental manipulations have modulated the presence of another person in the decision-making setting through variations in both the level of emphatic-related responses and the reputational value of the decisions.

The relationship between empathy and prosociality has been well established by behavioral and brain studies. There is evidence that relates prosocial behavior and emotional resonance in children $5,15,16$, as well as cooperation rates and empathetic perspective-taking in women ${ }^{17,18}$. Moreover, the perceptionaction model proposed empathy as an important factor for explaining altruistic behavior ${ }^{19}$. Neuroimaging evidence shows that in experimental tasks that involve observing others in pain, there is brain activity that overlaps with areas that are traditionally related to decision-making tasks. These structures include brain areas such as the ventromedial prefrontal cortex (vmPFC), anterior insula (Al) and midcingulate cortex (MCC, commonly referred to as the dorsal anterior cingulate cortex, see ${ }^{20}$ ). Specifically, Al activity has been related to being more prosocial towards people perceived as victims of pain ${ }^{6}, \mathrm{Al}$ and $\mathrm{MCC}$ activity has been related to helping behavior ${ }^{8}$, and vmPFC activation to others' suffering and involved in charity donations ${ }^{9}$. There is also electroencephalographic (EEG) evidence showing oscillatory activity related to the presence of others with different degrees of empathy involvement. For instance, increased frontal theta activity has been related to observing others in pain (compared to neutral stimuli) as well as to one's own unpleasant feelings ${ }^{21,22}$. Frontal theta activity has also been related to self-reflexive thinking within social interactions ${ }^{23}$ and the prediction of others' decisions ${ }^{16,24}$. Taken together, these data support the relevance of the presence of others on people's decision making and that there are different ways in which a given other sneaks into the variables that a decision-maker takes into account while deciding.

Prosocial decisions can also have indirect benefits for decision-makers in the form of reputation. In this respect, there is evidence showing the tendency of people to cooperate (or defeat) with those who cooperated (or defeat) with them in the past (known as direct reciprocity or DR) ${ }^{10}$ and to help those who help others (known as indirect reciprocity or IR) ${ }^{25,26}$. This latter form of reciprocity (IR) is proposed as the reason why it would pay to develop a reputation of being prosocial. Thus, the presence of an observer might influence prosociality related to the potential benefits that this behavior provides in the form of reputation and eventual reciprocity. For instance, when dictators make their offers facing eyespots on the screen as an indicator of observability, they increase their offers over the expected ${ }^{27}$. Moreover, in an 
experiment performed by ${ }^{28}$, participants who performed the dictator game (DG) and the ultimatum game (UG) proposed significantly fairer offers in the UG than in the DG condition. These fairer offers within the UG setting were accompanied by higher activation in the right dorsolateral prefrontal cortex (dIPFC) and the vmPFC. The involvement of these areas suggests predictions about possible rejection by recipients 29,30 and the inhibition of immediate self-oriented responses to obtain higher final payoffs ${ }^{31-33}$. Brain models of social cognition have shown different networks related to activity in the TPJ and dmPFC related to inferring another's mental states and the amygdala and vmPFC related to the affective value of social behavior ${ }^{34-42}$. Recent evidence has shown the relevance of the MCC in social cognition, particularly given its connectivity to the listed regions and its modulation to other-oriented information 34,43 .

In this context, the present study aims to assess, through a slightly modified version of the DG, the impact that the presence of another person has on the decision process of a person who has to choose distributions of money for him/herself and for another player. We studied the behavior and brain activity of adult individuals while choosing within three different scenarios: when the decisions were anonymous (control condition, CC), when participants were observed by another person (observer condition, $\mathrm{OC}$ ), and when the decisions were in the context of another person who is in need (empathy condition, EC). These contextual manipulations aimed to modulate the intensity of the empathy concerns triggered by the presence of another person within the decision setting, or, in other words, the "empathy saliency" of someone in need. We hypothesized that the presence of another person would increase prosocial decisions and that this increase would be higher when the other person is associated with others in need $(E C)$. At the brain level, we hypothesized that the increase in prosocial decisions would correlate with frontal theta activity as a marker of empathy saliency. The results showed that EC, but not OC, increased prosocial behavior compared to $\mathrm{CC}$. Brain activity showed an increase in frontocentral theta-oscillatory activity when participants faced unequal advantageous distributions to the other player within the EC. This brain activity can be interpreted as the marker of the social encoding related to the presence and attention given to the other person within the decision process.

\section{Material And Methods}

\section{Participants}

Thirty-two healthy participants (13 women) with normal or corrected-to-normal vision, without a history of neurological or psychiatric diagnosis, performed the experimental task. Their age range was between 19 and 23 years old $(M=21.4, S D=1.3)$. Participants were randomly assigned to one of the two experimental conditions (see experimental task below). The experimental procedures were performed in accordance with institutional guidelines and were approved by the Ethical Committee of the Pontificia Universidad Católica de Chile. All participants gave their written informed consent. All experiments were carried out at the Laboratorio de Neurociencia Social y Neuromodulación of the Centro de Investigación en Complejidad Social (neuroCICS), Universidad del Desarrollo, Santiago, Chile. 


\section{Power and sample size}

To calculate the minimum sample size and the power of the current study, we used the behavioral effect as the primary outcome. A similar study related to the effect of the presence of others during socioeconomic decision making ${ }^{27}$ shows an effect size of $\eta^{2}=0.149$, which is a large effect ${ }^{44}$. Taking into account publication bias, we set an intermediated effect size of $\eta^{2}=0.1$. Thus, for the between-within factor interaction in a $2 \times 2$ mixed ANOVA with a power of $(1-\beta)=0.95$ and a significance level of $\alpha=0.05$, the minimum sample size to find the expected effect was $n=32$.

\section{Experimental task}

The experimental task consisted of a modified version of the DG, in which participants had to choose between two possible allocations of money for themselves and for another player (hereinafter the other). Within the two possible choices, there was always one that gave participants the option of being prosocial. In the case of this task, a prosocial decision is defined as choosing the option that provides higher incomes to the other player regardless of the consequences for the participant. All participants were informed that they would receive an amount of money at the end of the experiment, which would correspond to the sum of two factors. One is composed of one of their own decisions (one trial selected at random), and the other corresponds to the decision made by a previous player, also selected at random. In the latter case, the amount corresponds to the money that a previous participant paid to the other player during his/her performance of the task. This design has the aim of highlighting that decisions have real consequences for both the participants themselves and another person.

The sample was randomly assigned to one of the two groups: the empathy group and the observer group. For both groups, participants first performed a round of the task as a control condition, in which their decisions were told to be anonymous. After this first round, participants assigned to the Empathy group performed a second round of the task under EC. The EC is the same task as in the CC, but at this time, participants were told that this part of the experiment was part of a larger study that involved donation of money to a real charity institution but that these donations did not depend on the specific decision of the participants. They were informed that, under this condition, the decisions were anonymous. It is important to note that, at this point, all the participants received the explicit clarification that the amount of money that they allocated was not a direct donation to the charity institution. No further information was given about the charity institution, and a blurred picture of a child who would benefit from this donation was shown to the participants during the rest of the experimental task. Participants assigned to the observer group, after playing the $\mathrm{CC}$, played a round of the task under the $\mathrm{OC}$. The $\mathrm{OC}$ is the same task as in the CC, but at this time, participants were observed by another researcher who is part of the research team 
while they were playing, so their decisions were no longer anonymous. They were informed that the observer was a sociologist who conducted the observation as part of another study. A blurred picture of the observer was shown to the participants for the rest of the experimental task (as in the EC). Moreover, a confederate playing the role of a sociologist entered the experimental room several times during the experiment, taking notes and observing the participants while playing.

The decision task consisted of the selection of one within two distributions of money that were presented in the higher and lower parts of the screen. These distributions or allocations were presented separately for 1500 ms each, as shown in Figure 1. Both allocations involved money for the participant and for another player. The other player was identified as a future participant in the experiment. The amounts for participants were presented on the left side of the screen in yellow, and the amounts to the other player were presented on the right side of the screen in blue (see figure 1). The amounts were presented for 1500 $\mathrm{ms}$, and a visual cue (green fixation cross) indicated to the participants that the decision could be delivered. During all conditions, participants faced three types of choices or cases that were randomly presented: others' advantageous inequity (OAI), others' disadvantageous inequity (ODI) and altruistic inequity (ALT) (see Figure 2). In the ALT choices, the prosocial option involves a personal cost to the participant. In the OAl cases, there is no such conflict as in the ALT case, given that the prosocial option involves higher earnings for the other participant but no personal costs to the own players. In the ODI cases, the prosocial option prevents the other participant from obtaining lower earnings with no personal costs to the player. Finally, participants were told that sometimes the game would choose the opposite option to the one chosen by them (e.g., if the participant chose the prosocial option, then the nonprosocial option would be displayed as the chosen option). If they want to return to their original option, they will be punished in that trial by losing a fixed amount of USD $\$ 5$, but if they want to keep the "error", then that choice (the opposite of the one they wanted) will be considered. This was used as a way to confirm the strength of participants' decisions. At the end of the experimental task, all participants received a payment from one of the played trials (selected at random) plus what a previous participant left to them.

\section{EEG}

Brain activity was recorded from 64 scalp electrodes using a Brain Vision amplifier system (BrainProducts, Germany). BrainVision Recorder was used to record brain activity (electrode impedance $<5 \mathrm{k} \Omega, 0.15-500 \mathrm{~Hz}, 1000$ samples/s). All recorded EEG epochs were individually checked for artifacts by visual inspection. Artifacts were first automatically detected using a threshold of $150 \mathrm{uV}$ and a power spectrum greater than $2 \mathrm{std}$. dev. for more than $10 \%$ of the frequency spectrum ( 1 to $30 \mathrm{~Hz}$ ). Blinking was extracted from the signal by means of ICA. Trials that included artifacts detected automatically and confirmed by visual inspection of the signal were eliminated. The artifact-free EEG material was recomputed to average reference and digitally bandpassed filtered to $0.1-45 \mathrm{~Hz}$. Whole power distribution was computed using Wavelet transform, with a 5-cycle Morlet wavelet, in a -1.5 to $1.5 \mathrm{~s}$ 
window around the onset of the second offer. For all analyses, we used the $\mathrm{dB}$ of power related to the baseline ( 15 seconds acquired at the beginning of each block).

\section{Statistical Analysis}

We used the Kolmogorov-Smirnoff test for normality. When the data did not meet the normal assumption, we used nonparametric tests. For the EEG statistical analysis, we first fitted a general linear model (GLM) of the power of the oscillatory activity per trial in each participant (first-level analysis, see ${ }^{45-48}$ ) using the following equation:

Power(t,f) $=\beta_{1}+\beta_{2} \Delta \mathrm{A} 1+\beta_{3} \Delta \mathrm{A} 2+\beta_{4} \Delta \mathrm{A} 1 * \Delta \mathrm{A} 2+\beta_{5} \mathrm{~T}+\beta_{6} \Delta \mathrm{A} 1 * \mathrm{~T}+\beta_{7} \Delta \mathrm{A} 2{ }^{*} \mathrm{~T}+\beta_{8} \Delta \mathrm{A} 1 * \Delta \mathrm{A} 2 * \mathrm{~T}$

where $\beta_{1}$ is the intercept, $\beta_{2}$ is the slope (coefficient) of the variable $\Delta \mathrm{A} 1$ (differences between the allocation for the player and the other in the first presented distribution), $\beta_{3}$ is the slope of the difference between the allocations for the player and the other in the second presented distribution $(\triangle A 2)$, and $\beta_{4}$ is the slope of the differences between the allocation for the player and the other in both offers (note that this regressor takes values other than zero only in the ALT case). Additionally, we added a regressor for the experimental condition ( $T$, which takes the value 1 when the decision is made during the experimental manipulation, observer or empathy conditions, and 0 in the control condition) with its respective slope $\left(\beta_{5}\right)$, together with the interactions between the experimental condition and the other regressors $(\triangle A 1 * T$, $\triangle \mathrm{A} 2^{*} \mathrm{~T}$ and $\triangle \mathrm{A} 1^{\star} \Delta \mathrm{A} 2^{*} \mathrm{~T}$ ). Then, we obtained a $3 \mathrm{D}$ matrix of the normalized $\beta$-values estimated (electrode, time, frequency, $\beta$-value/standard error) for each regressor and participant. We then explored for differences between groups (observer and empathy) and conditions (control and experimental) using the Wilcoxon test (second-level analysis). To correct for multiple comparisons in the time-frequency domain, we used the cluster-based permutation (CBP) test ${ }^{49}$. Briefly, in this method, the clusters of significant areas were defined by pooling neighboring sites (in the time-frequency chart and adjacent electrodes) that showed the same statistical effect (cluster threshold detection, CTD, uncorrected $p<.05$ ). The clusterlevel statistics were computed as the sum of the statistics of all sites within the corresponding cluster (e.g., Z value for Wilcoxon test). We evaluated the cluster-level significance under the permutation distribution of the cluster that had the largest cluster-level statistics. The permutation distribution was obtained by randomly permuting the original data (i.e., permuting a specific regressor per trial for withinsubject analyses or group labels for between-subject analyses). After each permutation, the original statistics test was computed (i.e., Wilcoxon test), and the cluster-level statistics of the largest resulting cluster were used for the permutation distribution. After 5000 permutations, the cluster-level significance 
for each observed cluster was estimated as the proportion of elements of the permutation distribution larger than the cluster-level statistics of the corresponding cluster.

\section{Results}

\section{Behavioral results}

To assess participants' decisions, a mixed ANOVA was conducted using case (type of offers, i.e., ALT, OAI or $\mathrm{ODI}$ ) and condition (control or empathy/observer) as independent variables and prosocial responses as the dependent variable. The variable group (i.e., observer or empathy) was used as a between subjects factor.

Table 1

Mixed ANOVA with case, condition and group as independent variables and responses as the dependent variable.

\begin{tabular}{|lllllll|}
\hline & $\begin{array}{l}\text { Sum of } \\
\text { Squares }\end{array}$ & df & $\begin{array}{l}\text { Mean } \\
\text { Squares }\end{array}$ & F & p & $\eta^{2}$ \\
\hline Condition & 0.900 & 1 & 0.900 & 17.857 & $<.001$ & 0.31 \\
Residual & 1.966 & 39 & 0.050 & & & \\
Case & 23.473 & 2 & 11.736 & 87.905 & $<.001$ & 0.693 \\
Residual & 10.414 & 78 & 0.134 & & & \\
\hline Condition * Case & 0.224 & 2 & 0.112 & 3.950 & 0.023 & 0.092 \\
Residual & 2.216 & 78 & 0.028 & & & \\
$\begin{array}{l}\text { Condition * Case * Group (between } \\
\text { subjects) }\end{array}$ & 0.302 & 2 & 0.151 & 6.004 & 0.004 & 0.06 \\
Residual & 1.913 & 76 & 0.027 & & & \\
\hline
\end{tabular}

Significant main effects of case and condition (see Table 1) together with the interaction between condition and case were found. This suggests that participants tended to cooperate differently depending on the case. A between-subjects interaction with condition and case was also found.

Participants in both groups tended to be less prosocial in ALT cases than in the other two cases (post hoc ALT-OAl: $p($ bonf $)<0.001, t=-7.891$, Cohen's $d=-2.087$ ). This means that when the prosocial options involved personal costs, participants decided to choose it less than when there were no costs associated with this choice. Interestingly, participants decided to be less prosocial in OAI cases than in ODI cases (post hoc ODI-OAI: $p($ bonf $)<0.001, t=3.230$, Cohen's $d=0.839$ ), even though in both cases, there were no personal costs involved in their decisions. This means that participants decide to be more prosocial when this prevents other individuals from earning less than them but were less prosocial when the other participants could earn more than them in the trial (see figure $3 \mathrm{~A}$ ). 
The separate analysis of both groups shows an effect of the EC but not of the OC (see figure 3). Within the observer group (figure $3 \mathrm{C}$ ), there was no effect of the experimental intervention on the prosociality rates (post hoc control (c) ALT - experimental (e) ALT: $p$ (bonf)=1, $t=0.384$; post hoc cODI-eODI: $p($ bonf) $=1$, $t=-6.22$; post hoc cOAl-eOAl: $p$ (bonf) $=0.9, t=-1.38$ ). Within the Empathy group (figura $3 \mathrm{~B}$ ), there was an effect of the experimental intervention in both ALT and OAI cases where the prosociality rates increased relative to the control condition (post hoc cALT-eALT: $p$ (bonf)<0.001, $t=-6.153$, cOAl-eOAl: $p$ (bonf)<0.001, $t=-4.713$ ). There were no differences between the ODI cases (cODI-eODI: $p$ (bonf)=1, $t=-6.95)$. The prosociality rates in the ODI case were high in the control condition across both experimental interventions. The between-subject effects show no differences in the cooperation rates between the two control conditions in all cases $(p($ bonf $)=1$ for the three cases).

To closely observe the effect of the experimental intervention, particularly in the Empathy group, a temporal analysis of the prosocial choices was conducted (see figure 3D). The control and experimental conditions were divided into 3 blocks of trials each to observe in more detail the changes in prosocial choices across the experimental task. The variable blocks and cases were used as independent variables, and prosocial responses were used as dependent variables. Within the Empathy group (figure 3E), the results confirm the effect of the experimental intervention (post hoc block3-block4: $p$ (bonf)<0.001 $t=-6.008$ ) but not in the Observer group (figure 3F) ( $($ bonf $=1)$. Prosocial choices within the empathy group remained stable across the last 3 blocks of the EC (post hoc block4-block5: $p($ bonf)=1; block5block6: $\mathrm{p}($ bonf $)=1)$.

As mentioned previously, during the experiment, $30 \%$ of trials indicated that the game chose the opposite option as the one chosen by participants, who had the option to return to their original choice at a monetary cost. We analysed whether there were differences between the control and experimental conditions in the proportion in which participants maintained or changed their options given their initial choice. The dependent variable for the analysis was the proportion in which subjects maintained a prosocial choice (this means, if they chose the prosocial choice, the game inverted their decisions and then participants returned to their original prosocial election) and the proportion in which participants changed their original nonprosocial choice to the prosocial choice selected by the game.

A repeated-measures ANOVA was conducted using group, experimental conditions and offers as independent variables and the proportion of confirmatory prosocial choices as the dependent variable. The results (see figure 4 ) show an effect of prosociality $\left(p<0.001, F=19.572, \eta^{2}=0.177\right)$ on the interaction between group and prosociality $\left(p=0.046, F=4.263, \eta^{2}=0.038\right)$ and on the interaction between condition, group and prosociality $\left(p=0.003, F=10.098, \eta^{2}=0.028\right)$.

Post hoc analyses show that participants within the observer group tend to confirm their nonprosocial choice more than their prosocial choice in both control and experimental conditions (post hoc pro-nonpro $p($ bonf $)<0.001, t=4.527$ ). This means that when participants chose the nonprosocial option but the opposite was presented to them, they returned to their original choice in a higher proportion than when they chose the prosocial option in the first place. In the empathy group, there were no differences in the 
proportion of choices between the control and experimental conditions. The between-subject effect shows that there were no differences between the groups in either condition.

\section{Brain results}

A time-frequency analysis was conducted to observe the effect of the differences in the spectral activity, particularly in the theta-band oscillatory activity, relative to the unequal monetary distributions during the $\mathrm{ODI}$ and OAI cases. As mentioned above, there is consistent evidence showing that theta oscillatory activity is related to self-inhibitory activity and the encoding of social cues during decision processes. This analysis was conducted given the behavioral differences found between the ODI and OAI cases within the empathy group. These differences show that while participants decided to be similarly prosocial in the ODI cases in both control and experimental conditions, there were higher rates of prosociality in the OAl experimental treatment. This is interesting given that, in both cases, the players choose between options with same earnings for themselves, but while in the ODI case the prosocial choice prevents the other player from receiving less than them, in the OAl case the prosocial choice involves that the other player receives more than them. Thus, we first fitted a GLM of the power of the oscillatory activity per trial during the presentation of the second offer. As a regressor, we used the differences in the earnings for participants themselves and for the other player in the first and the second offers separately and the interaction between them. Note that the interaction captures the variance of the signal given by ALT cases (see method); thus, the other regressors capture the variance given by the other cases (ODI and OAI). Specifically, these regressors capture the difference between ODI and OAI when no equal offers are presented in the first distribution $(\triangle A 1)$ or in the second distribution ( $\triangle A 2)$. We mainly focused on exploring the different modulations in these regressors for experimental conditions (using the interaction: $\triangle \mathrm{A} 1^{\star} \mathrm{T}$ and $\triangle \mathrm{A} 2^{\star} \mathrm{T}$, see methods). For the contrast of the second-level analysis, we pooled both regressors $(\triangle \mathrm{A} 1 * \mathrm{~T}$ and $\triangle \mathrm{A} 2 * \mathrm{~T})$ to capture the variance related to the decision processing when facing these cases under experimental conditions. Thus, we explored for differences between groups and conditions using the Wilcoxon and CBP tests (see Methods for more details).

Based on prior research ${ }^{50}$, we first explored modulation in frontal electrodes $(C z, F C z, C 1, C 2, F C 1, F C 2$, depicted in the white rectangle in Figure $5 \mathrm{E}$ ). For the Empathy group, we found a negative modulation of theta/alpha activity approximately $300 \mathrm{~ms}$ after the second offer (Figure 5A). In other words, there was an increase in theta/alpha activity when participants faced OAI cases (in comparison with ODI cases) in the experimental (empathy) condition. For the observer group, we did not find any significant modulation (Figure 5B). These results led to a significant difference in frontocentral theta oscillatory activity modulation between the empathy and observer groups during the experimental intervention (Figure $5 \mathrm{C}$ and $\mathrm{E}$ ). These results indicate that $\mathrm{EC}$, but not $\mathrm{OC}$, led to an increase in frontocentral theta oscillatory activity when participants were exposed to cases in which the other player could earn more than themselves.

These results were corroborated using a whole scalp analysis without a priori assumptions about the localization of the brain modulation between groups (Figure 5D). This analysis shows the emergence of a 
consistent cluster that overlaps with the activity found in the preceding analysis. Interestingly, the analysis also showed another modulation in theta/alpha oscillatory activity when participants watched the cue that indicated that the decision had to be delivered (Figure 5F). Source analysis indicated that theta modulations after the second offer are located in the MCC and right superior frontal gyrus (r-SFG), while the modulations after the response cue are located in the left middle frontal gyrus (I-MFG, or dIPFC) (Figure 5G).

Finally, we explored whether theta/alpha activity after both the second offer and the response cue were related to the rate of prosocial choices. For this, we correlated the normalized b-value per subject in both the r-SFG after the second offer and in the I-MFG after the response cue with the rate of prosocial decisions in OAI cases during the experimental condition. Interestingly, the Spearman partial correlation showed that prosocial decisions in OAl cases during treatment correlated with theta activity in the r-SFG $(r=-0.57, p=0.02, n=16)$ and not with theta activity in the I-MFG $(r=0.3, p=0.2, n=16)$ but only for the observer group (empathy group: abs $(r)<0.04, p>0.8$ ). To explore this with more detail, we carried out a robust linear regression for the rate of prosocial decisions in the OAI case for experimental conditions. In accordance with the preceding result, the relation between theta activity in the r-SFG and prosocial decisions differed between the observer and empathy groups theta activity (see Table 2).

Table 2

Robust linear regression of the prosocial decision in the OAl case during experimental conditions for both groups.

\begin{tabular}{|lllll|}
\hline & beta & S.E & t-Value & p-Value \\
\hline Int & 0.39 & 0.08 & 4.4 & 0.0001 \\
\hline Theta (I-MFG) & 0.03 & 0.03 & 1.05 & 0.3 \\
\hline Theta(r-SFG) & -0.13 & 0.05 & -2.3 & 0.027 \\
\hline Observer Condition (OC) & 0.14 & 0.1 & 1.4 & 0.17 \\
\hline Theta (r-SFG)*OC & 0.21 & 0.09 & 2.2 & 0.035 \\
\hline Theta (I-MFG)*OC & -0.02 & 0.07 & -0.36 & 0.7 \\
\hline OAl control condition & 0.64 & 0.12 & 5.2 & $<0.0001$ \\
\hline
\end{tabular}

Taken together, these results show that the empathy intervention increases prosocial decisions modulating frontal theta activity when evaluating the different options and when making the decision. The observer intervention did not modulate the prosocial decision on the whole group of subjects, but those subjects that increased their prosocial decision demonstrated a theta modulation that was similar to the one that the subjects in the empathy group showed.

\section{Discussion}


Based upon previous experimental results, we hypothesized that the presence of another person would increase prosocial decisions and that this increase would be higher when the other person is associated with others in need (EC). At the brain level, we hypothesized that the increase in prosocial decisions correlates with frontal theta activity as a marker of empathy saliency. The results showed a differential effect between the two experimental conditions (OC and EC) in both behavior and brain activity. While the empathy group showed an increase in prosocial decisions during the experimental condition associated with a modulation of frontal theta activity, there were no effects in the observer group. The brain activity and behavioral change found in the EC can be interpreted as an enhancement of the saliency of someone in need and/or an increase in social norm compliance.

Brain results show that the inclusion of someone in need had an effect in the way in which participants faced the decision with favorable inequity to the other player (OAI cases). Oscillatory activity shows that this experimental intervention increased frontal theta activity relative to the unequal favorable distribution to the other player. The source of this activity is compatible with the posterior part of the MCC and superior frontal gyrus. The MCC has been traditionally associated with several processes related to decision making, particularly error detection, self-monitoring, socially driven interactions and empathyrelated responses ${ }^{45,47,51-53}$. There are reports of a general role of the MCC in the processing of multimodal context-dependent events ${ }^{54,55}$, which has been specifically observed in pain-related experiments that modulate empathy responses ${ }^{56-61}$. Macaque studies, moreover, have shown that lesions to the anterior cingulate gyrus decreased the value that those animals give to social stimuli ${ }^{34}$ and that activity in this area is related to the observation of rewarding outcomes delivered to another animal 43. Human studies have shown that the MCC processes cues that inform the motivational state of another person, providing the interpretation that this area participates in the monitoring of others' behavior for learning about and from others ${ }^{34}$. Taking our results in context to the presented evidence, we can suggest that the frontal theta activity introduced by the empathy condition reflects the participants' detection of relevant social information given by the other person in need. Although the person in need in the EC does not receive a direct benefit from the participants' decisions, the recruitment of the preceding brain mechanism can increase the participants' prosocial decisions. It is important to note that this effect was not present during the $\mathrm{OC}$ although there is also another person present in the decision setting. Interestingly, participants who increased their prosocial choices in the OC showed a theta modulation similar to the EC. The stronger effect observed during EC can be due to emotion-related processing elicited by the inclusion of the other in need into the decision setting. Indeed, in accordance with our source analysis, the posterior part of the MCC has been extensively associated with empathic/emotional resonance $20,56,62$.

Another possible interpretation for the findings is that the inclusion of another person in need modulated a more general compliance-to-the-norm response. Subjects across both groups tended to be less prosocial when there were personal costs associated with the prosocial choice. This is in line with profuse evidence showing lower rates of prosocial decisions in the dictator game (DG) than in the ultimatum game $(U G){ }^{63-65}$. While in the former game there are no monetary incentives for prosociality, in 
the latter game subjects need to consider others' earnings to obtain higher outcomes. Our results show that participants tended to be more prosocial when this prevented other participants from earning less than them but were less prosocial when this led the other with higher earnings. Our brain results show an increase in frontal theta activity relative to the unequal favorable distribution to the other player. This kind of activity has been previously associated with fair behavior in the DG and has been interpreted as cognitive control and self-inhibition ${ }^{50,53}$. There is evidence showing medial-prefrontal activity related to rejection of unfair offers in the UG ${ }^{66}$ and specific insular activity related to unfair rejections to third parties. This frontal activity associated with cognitive control and inhibition is in line with evidence that shows the role of the dIPFC (sometimes with specific lateralization to the right hemisphere) related to the compliance of social norms, self-inhibition and cognitive control ${ }^{67}$. Following the preceding interpretation of frontal activity, theta activity in the EC might reflect a general response to adapt the behavior to a norm, in this case related to suppressing inequity-aversion responses when inequity was advantageous to the other player. There is evidence that social norms are reinforced by the presence of a third-party observer ${ }^{67}$. Previous evidence using social observation has shown effects in the way the brain encodes fairness, particularly in the context of social anxiety relative to the observation ${ }^{67}$. Notably, we found behavioral and brain effects only in the empathy group and not in the observer group. This suggests that the observer-experimental manipulation has to be conducted more strongly to produce more general brain and behavioral changes in the decision process. It can be suggested that future experimental designs include the measurement of personality traits to evaluate whether there are different sensibilities related to the influence of making decisions under observation, since we found a correlation between behavior and theta activity within the observer group.

We have discussed two possible interpretations of our brain and behavioral results, one related to the effect of empathy-related responses associated with a person in need and another related to a more general compliance-to-the-norm response. These two interpretations can be considered complementarity. Recently, fairness-related normative decision making in the context of social interaction has been analysed from the perspective of two complementary processes: an intuitive component and a deliberative one ${ }^{68}$. The intuitive component is related to the responses towards conflicts between selfinterests and a given social context; it is usually related to emotional factors and is reported as being the responsible component for altruistic punishment and general prosocial responses ${ }^{65,69-74}$. The deliberative component has been related to a reappraisal process of conflict evaluation between, for instance, unfairness-evoked aversive responses (norm enforcement) and self-interest concerns ${ }^{68}$. The brain areas related to this component are the MCC, dIPFC, vIPFC and dmPFC and are thought to contribute to the development of flexible strategies within decision-making settings. Although the source analyses have to be interpreted with caution, we can at least point out that the brain activity associated with the EC is in line with the idea of an early component associated with the inclusion of the "other" via the emotionally related component and that this response may have been reinforced by a normcompliance adjustment. 
Taken together, these results show that the inclusion of a person in need into the decision setting enhances prosocial behavior given by the inhibition of other-advantageous inequity aversion strategies. The modulation of theta brain activity indicated that these effects can be due to the enhancement of either empathy concerns or norm compliance. Due to the correlative nature of our experiment, it is necessary to inquire about the causal role of these possible brain and cognitive mechanisms by testing other experimental approaches, for example, using noninvasive brain stimulation.

\section{Declarations}

\section{Competing interests}

The authors report no biomedical financial interest or potential conflicts of interest.

\section{Author contributions}

C.L., V.L., P.B. designed the experiment; C.L., P.B. programmed the experiment; C.L., P.B., conducted the experiments; C.L., P.B., analysed the data; C.L., P.S-I., V.L., P.B., interpreted and discussed the results; C.L., P.S-I., V.L., P.B., wrote the manuscript.

\section{Funding}

This work was supported by the Chilean National Scientific and Technological Research Commission (CONICYT) (PCHA/National Doctoral Program/Grant 2016-21160667, FONDECYT 12211227; CONICYT PAI PAI77190047; EQM150076).

\section{References}

1. Balconi, M. \& Canavesio, Y. Prosocial attitudes and empathic behavior in emotional positive versus negative situations: brain response (ERPs) and source localization (LORETA) analysis. Cogn Process 14, 63-72 (2013)

2. Fehr, E. \& Fischbacher, U. The nature of human altruism. Nature 425, 785-791 (2003)

3. Hewig, J. et al. Why humans deviate from rational choice. Psychophysiology 48, 507-514 (2011) 
4. Waal, F. B. M. de. Putting the Altruism Back into Altruism: The Evolution of Empathy. Annu Rev Psychol 59, 279-300 (2008)

5. Williams, A., O'Driscoll, K. \& Moore, C. The influence of empathic concern on prosocial behavior in children. Front Psychol 5, 425 (2014)

6. Masten, C. L. \& Morelli, S. A. \& Eisenberger, N. I. An fMRI investigation of empathy for 'social pain' and subsequent prosocial behavior. Neuroimage 55, 381-388 (2011)

7. Morelli, S. A., Rameson, L. T. \& Lieberman, M. D. The neural components of empathy: Predicting daily prosocial behavior. Soc Cogn Affect Neur 9, 39-47 (2014)

8. Rameson, L. T., Morelli, S. A. \& Lieberman, M. D. The Neural Correlates of Empathy: Experience, Automaticity, and Prosocial Behavior. J Cognitive Neurosci 24, 235-245 (2012)

9. Hare, T. A., Camerer, C. F., Knoepfle, D. T., O’Doherty, J. P. \& Rangel, A. Value computations in ventral medial prefrontal cortex during charitable decision making incorporate input from regions involved in social cognition. J Neurosci Official J Soc Neurosci 30, 583-90 (2010)

10. Trivers, R. L. The Evolution of Reciprocal Altruism. Q Rev Biology 46, 35-57 (1971)

11. Steinbeis, N., Bernhardt, B. C. \& Singer, T. Impulse Control and Underlying Functions of the Left DLPFC Mediate Age-Related and Age-Independent Individual Differences in Strategic Social Behavior. Neuron 73, 1040-1051 (2012)

12. Baumgartner, T., Knoch, D., Hotz, P., Eisenegger, C. \& Fehr, E. Dorsolateral and ventromedial prefrontal cortex orchestrate normative choice. Nat Neurosci 14, 1468-1474 (2011)

13. Knoch, D., Pascual-Leone, A., Meyer, K. \& Treyer, V. \& Fehr, E. Diminishing Reciprocal Fairness by Disrupting the Right Prefrontal Cortex. Science 314, 829-832 (2006)

14. Campanha, C., Minati, L., Fregni, F. \& Boggio, P. S. Responding to Unfair Offers Made by a Friend: Neuroelectrical Activity Changes in the Anterior Medial Prefrontal Cortex. J Neurosci 31, 1556915574 (2011)

15. Edele, A., Dziobek, I. \& Keller, M. Explaining altruistic sharing in the dictator game: The role of affective empathy, cognitive empathy, and justice sensitivity. Learn Individ Differ 24, 96-102 (2013)

16. Rodrigues, J. \& Ulrich, N. \& Hewig, J. A neural signature of fairness in altruism: a game of theta? Soc Neurosci 10, 192-205 (2014)

17. Batson, C. D. \& Ahmad, N. Empathy-induced altruism in a prisoner's dilemma II: what if the target of empathy has defected? Eur J Soc Psychol 31, 25-36 (2001)

18. Graaff, J. V. der, Carlo, G., Crocetti, E., Koot, H. M. \& Branje, S. Prosocial Behavior in Adolescence: Gender Differences in Development and Links with Empathy. J Youth Adolescence 47, 1086-1099 ( 2017)

19. Decety, J. \& Grèzes, J. The power of simulation: imagining one's own and other's behavior. Brain Res 1079, 4-14 (2006)

20. Vogt, B. A. Midcingulate cortex: Structure, connections, homologies, functions and diseases. J Chem Neuroanat 74, 28-46 (2016) 
21. Han, S. Neurocognitive Basis of Racial Ingroup Bias in Empathy. Trends Cogn Sci 22, 400-421 ( 2018)

22. Mu, Y., Fan, Y., Mao, L. \& Han S. Event-related theta and alpha oscillations mediate empathy for pain. Brain Res 1234, 128-36 (2008)

23. Mu, Y. \& Han S. Neural oscillations involved in self-referential processing. Neuroimage $53,757-68$ ( 2010)

24. Billeke, P., Zamorano, F., Cosmelli, D. \& Aboitiz, F. Oscillatory Brain Activity Correlates with Risk Perception and Predicts Social Decisions. Cereb Cortex 23, 2872-2883 (2013)

25. Roberts, G. Evolution of direct and indirect reciprocity. Proc Royal Soc B Biological Sci 275, 173-179 (2008)

26. Alexander, J. C. The Social Requisites for Altruism and Voluntarism: Some Notes on What Makes a Sector Independent. Sociol Theor 5, 165 (1987)

27. Haley, K. J. \& Fessler, D. M. T. Nobody's watching? Subtle cues affect generosity in an anonymous economic game. Evol Hum Behav 26, 245-256 (2005)

28. Spitzer, M., Fischbacher, U., Herrnberger, B., Grön, G. \& Fehr, E. The Neural Signature of Social Norm Compliance. Neuron 56, 185-196 (2007)

29. Kringelbach, M. L. The human orbitofrontal cortex: linking reward to hedonic experience. Nat Rev Neurosci 6, 691-702 (2005)

30. Doherty, O. J. \& Abstract reward and punishment representations in the human orbitofrontal cortex. Nat Neurosci 4, 95-102 (2001)

31. Sanfey, A. G., Rilling, J. K., Aronson, J. A. \& Nystrom, L. E. \& Cohen, J. D. The Neural Basis of Economic Decision-Making in the Ultimatum Game. Science 300, 1755-1758 (2003)

32. Aron, A. R., Robbins, T. W. \& Poldrack, R. A. Inhibition and the right inferior frontal cortex. Trends Cogn Sci 8, 170-177 (2004)

33. Miller, E. K. \& Cohen, J. D. An integrative theory of prefrontal cortex function. Annu Rev Neurosci 24, 167-202 (2001)

34. Apps, M. A. J., Rushworth, M. F. S. \& Chang, S. W. C. The Anterior Cingulate Gyrus and Social Cognition: Tracking the Motivation of Others. Neuron 90, 692-707 (2016)

35. Apps, M. A. J. \& Tsakiris, M. Predictive codes of familiarity and context during the perceptual learning of facial identities. Nat Commun 4, 2698 (2013)

36. Frith, C. D. \& Frith, U. The Neural Basis of Mentalizing. Neuron 50, 531-534 (2006)

37. Aboitiz, P. Soto-lcaza, F. \& Billeke, P. Development of social skills in children: neural and behavioral evidence for the elaboration of cognitive models. Front Neurosci-switz 9, 333 (2015)

38. Billeke, P., Soto-Icaza, P., Aspé-Sánchez, M. \& Villarroel, V. \& Rodríguez-Sickert, C. Neuroscience and Social Science, The Missing Link. 21-45 (2017) doi:10.1007/978-3-319-68421-5_2

39. Billeke, P. Soto-Icaza, P. \& Handbook of Social Behavior and Skills in Children. Autism Child Psychopathol Ser 47-62 (2017) doi:10.1007/978-3-319-64592-6_4 
40. Hampton, A. N. \& Bossaerts, P. \& O'Doherty, J. P. Neural correlates of mentalizing-related computations during strategic interactions in humans. Proc National Acad Sci 105, 6741-6746 ( 2008)

41. Lee, B. T. et al. Neural correlates of affective processing in response to sad and angry facial stimuli in patients with major depressive disorder. Prog Neuro-psychoph 32, 778-85 (2007)

42. Lee, D. \& Seo, H. Neural Basis of Strategic Decision Making. Trends Neurosci 39, 40-48 (2016)

43. Chang, S. W. C. \& Gariépy, J. F. \& Platt, M. L. Neuronal reference frames for social decisions in primate frontal cortex. Nat Neurosci 16, 243-250 (2012)

44. Cohen, J. Statistical Power Analysis for the Behavioral Sciences. (1988) doi: $10.4324 / 9780203771587$

45. Billeke, P. et al. Paradoxical Expectation: Oscillatory Brain Activity Reveals Social Interaction Impairment in Schizophrenia. Biol Psychiat 78, 421-431 (2015)

46. Larrain-Valenzuela, J. et al. Theta and Alpha Oscillation Impairments in Autistic Spectrum Disorder Reflect Working Memory Deficit. Sci Rep-uk 7, 14328 (2017)

47. Figueroa-Vargas, A. et al. Frontoparietal connectivity correlates with working memory performance in multiple sclerosis. Sci Rep-uk 10, 9310 (2020)

48. Vargas, P. \& Aboitiz, L. Soto-Icaza, F. \& Billeke, P. Beta oscillations precede joint attention and correlate with mentalization in typical development and autism. Cortex 113, 210-228 (2019)

49. Maris, E. \& Oostenveld, R. Nonparametric statistical testing of EEG- and MEG-data. J Neurosci Meth $164,177-190$ (2007)

50. Billeke, P. et al.. Someone has to give in: theta oscillations correlate with adaptive behavior in social bargaining. Soc Cogn Affect Neur 9, 2041- 2048 (2014)

51. Lavin, C. et al. The anterior cingulate cortex: an integrative hub for human socially-driven interactions. Front Neurosci-switz 7, 64 (2013)

52. Billeke, P. et al. Human Anterior Insula Encodes Performance Feedback and Relays Prediction Error to the Medial Prefrontal Cortex. Cereb Cortex 30, 4011-4025 (2020)

53. Zamorano, F. et al. Lateral Prefrontal Theta Oscillations Reflect Proactive Cognitive Control Impairment in Males With Attention Deficit Hyperactivity Disorder. Frontiers Syst Neurosci 14, 37 (2020)

54. Downar, J., Crawley, A. P., Mikulis, D. J. \& Davis, K. D. The Effect of Task Relevance on the Cortical Response to Changes in Visual and Auditory Stimuli: An Event-Related fMRI Study. Neuroimage 14, 1256-1267 (2001)

55. Downar, J., Crawley, A. P., Mikulis, D. J. \& Davis, K. D. A Cortical Network Sensitive to Stimulus Salience in a Neutral Behavioral Context Across Multiple Sensory Modalities. J Neurophysiol 87, 615-620 (2002)

56. Singer, T. et al. Empathy for Pain Involves the Affective but not Sensory Components of Pain. Science $303,1157-1162(2004)$ 
57. Singer, T. et al. Empathic neural responses are modulated by the perceived fairness of others. Nature 439, 466-469 (2006)

58. Decety, J. \& Jackson, P. L. A Social-Neuroscience Perspective on Empathy. Curr Dir Psychol Sci 15, 54-58 (2006)

59. Jackson, P. L., Brunet, E. \& Meltzoff, A. N. \& Decety, J. Empathy examined through the neural mechanisms involved in imagining how I feel versus how you feel pain. Neuropsychologia $44,752-$ 761 (2006)

60. Jackson, P. L. \& Meltzoff, A. N. \& Decety, J. How do we perceive the pain of others? A window into the neural processes involved in empathy. Neuroimage 24, 771-779 (2005)

61. Lamm, C. \& Decety, J. \& Singer, T. Meta-analytic evidence for common and distinct neural networks associated with directly experienced pain and empathy for pain. Neuroimage 54, 2492-2502 (2011)

62. Watson, J. C. \& Greenberg, L. S. The Social Neuroscience of Empathy. 125-138 (2009) doi:10.7551/mitpress/9780262012973.003.0011

63. Moore, L., Sugiyama, T. \& Grigaityte, K. Christov-, \& lacoboni, M. Increasing generosity by disrupting prefrontal cortex. Soc Neurosci 12, 174-181 (2016)

64. Yamagishi, T. et al. Cortical thickness of the dorsolateral prefrontal cortex predicts strategic choices in economic games. Proc National Acad Sci 113, 5582-5587 (2016)

65. Santamaría-García, H. et al. Explicit and implicit markers of fairness preeminence in criminal judges. Sci Rep-uk 11, 17599 (2021)

66. Civai, C. \& Rumiati, C. Corradi-Dell'Acqua, R. I. \& Fink, G. R. Disentangling self- and fairness-related neural mechanisms involved in the ultimatum game: an fMRI study. Soc Cogn Affect Neur 8, 424431 (2013)

67. Peterburs, J. et al. Processing of fair and unfair offers in the ultimatum game under social observation. Sci Rep-uk 7, 44062 (2017)

68. Feng, C., Luo, Y. J. \& Krueger, F. Neural signatures of fairness-related normative decision making in the ultimatum game: A coordinate-based meta-analysis: Neural Signatures of Decision Making in UG. Hum Brain Mapp 36, 591-602 (2014)

69. Haidt, J. The emotional dog and its rational tail: A social intuitionist approach to moral judgment. Psychol Rev 108, 814-834 (2001)

70. Roch, S. G., Lane, J. A. S., Samuelson, C. D., Allison, S. T. \& Dent, J. L. Cognitive Load and the Equality Heuristic: A Two-Stage Model of Resource Overconsumption in Small Groups. Organ Behav Hum Dec 83, 185-212 (2000)

71. Sanfey, A. G. \& Chang, L. J. Multiple Systems in Decision Making. Ann Ny Acad Sci 1128, 53-62 ( 2008)

72. Zaki, J. \& Mitchell, J. P. Equitable decision making is associated with neural markers of intrinsic value. Proc National Acad Sci 108, 19761-19766 (2011) 
73. Scheele, D. et al. Oxytocin Modulates Social Distance between Males and Females. J Neurosci 32, 16074-16079 (2012)

74. Zaki, J. \& Mitchell, J. P. Intuitive Prosociality. Curr Dir Psychol Sci 22, 466-470 (2013)

\section{Figures}

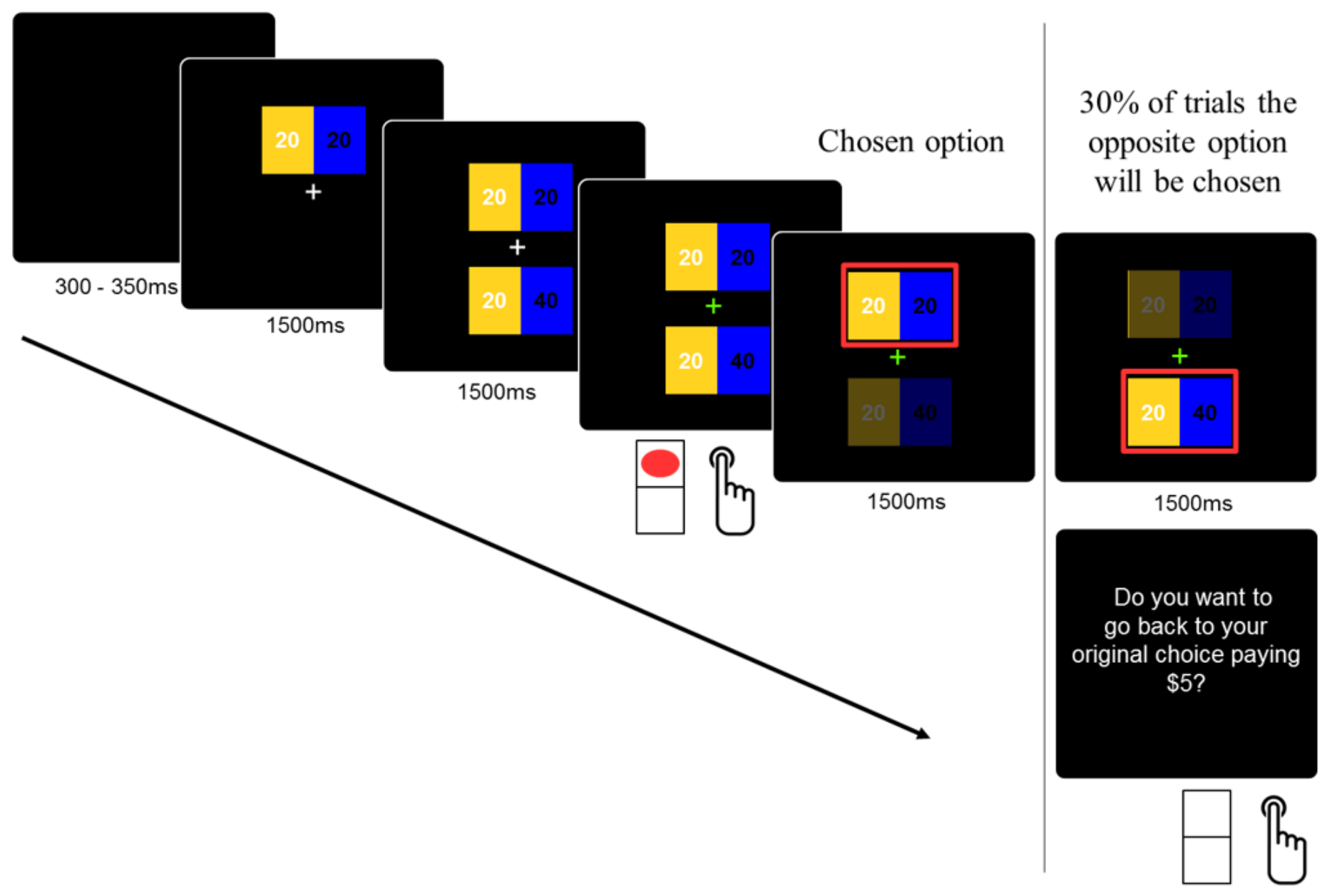

\section{Figure 1}

Experimental task, control condition. The decision task consisted of the selection of one within two distributions of money that were presented in the higher and lower parts of the screen. Both allocations involved money for the participant and for another player. The other player was identified as a future participant in the experiment. Participants had to press the bottom up or down according to their choice on a normal computer keyboard. 


\begin{tabular}{|c|c|c|c|c|}
\hline & \multicolumn{2}{|c|}{ Option 1 } & \multicolumn{2}{c|}{ Option 2 } \\
\hline $\begin{array}{c}\text { Other's } \\
\text { advantageous } \\
\text { inequity (OAI) }\end{array}$ & Low-pay & Low-pay & Low-pay & High-pay \\
\hline $\begin{array}{c}\text { Other's } \\
\text { disadvantageous } \\
\text { inequity (ODI) }\end{array}$ & High-pay & High-pay & High-pay & Low-pay \\
\hline $\begin{array}{c}\text { Altruistic } \\
\text { (ALT) }\end{array}$ & Low-pay & High-pay & High-pay & \\
\hline
\end{tabular}

\section{Figure 2}

Structure of distributions presented to participants during the experimental task 


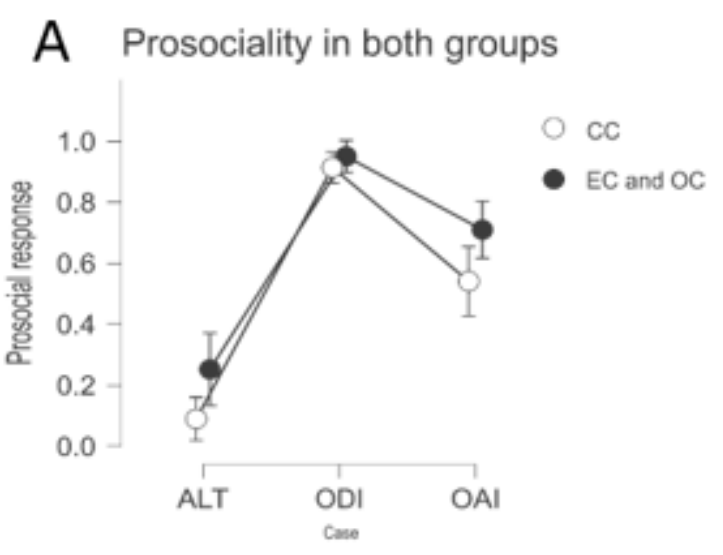

B Prosociality in the Empathy group

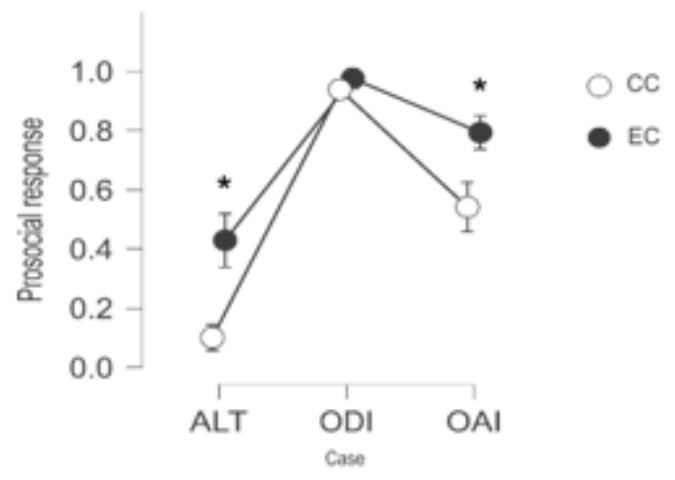

C Prosociality in the Observer group

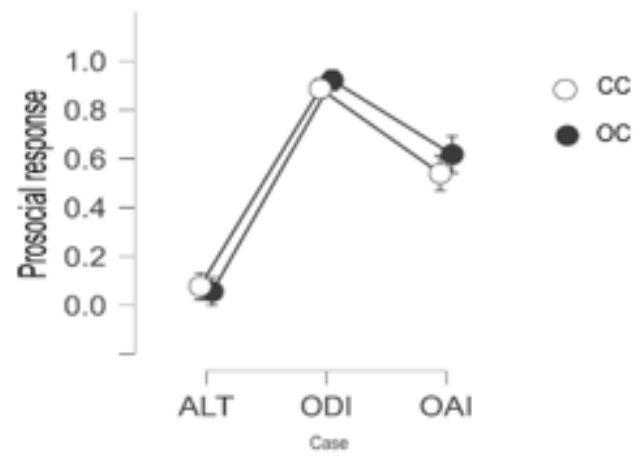

D Prosociality in both groups by blocks

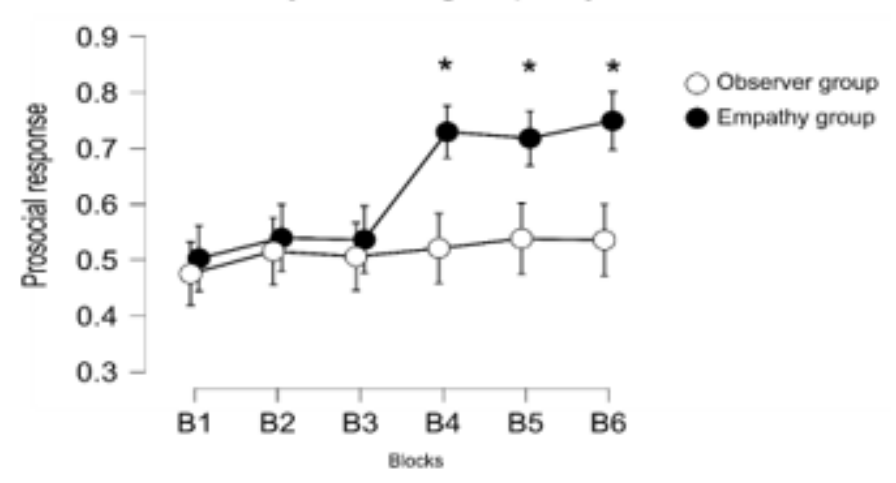

E Prosociality in the Empathy group by blocks

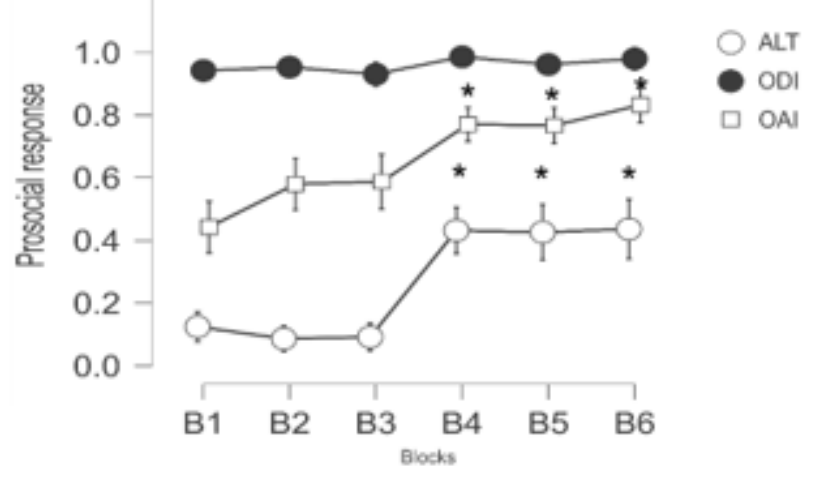

F Prosociality in the Observer group by blocks

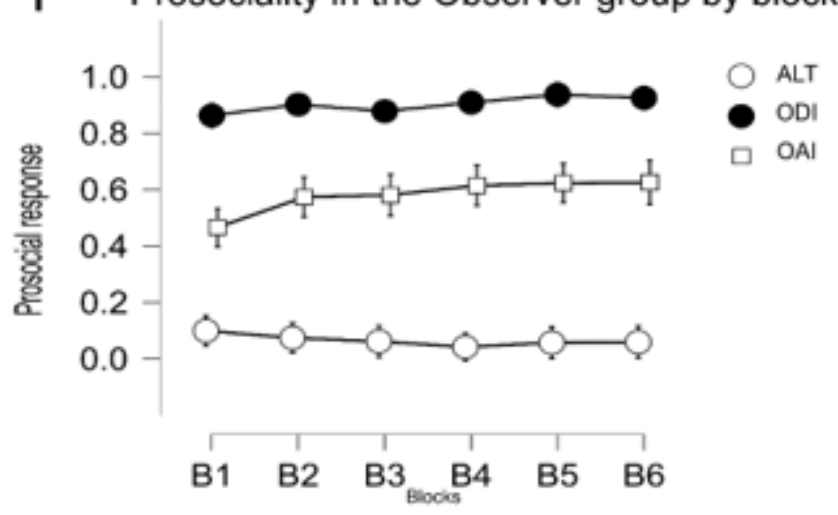

\section{Figure 3}

(A) Means and standard deviation of the cooperation rates across each 3 cases by both groups collapsed. (B) Means and standard deviation of the cooperation rates across the 3 cases in the observer group. (C) Means and standard deviation of the cooperation rates across the 3 cases in the Empathy group. (D) Means and standard deviation of the prosocial choices across 6 blocks of trials during the experiment. Block 4 is the first block of the experimental condition across both interventions. (E) Means and standard deviation of the cooperation rates by case across 6 blocks of trials in the Empathy group. (F) Means and standard deviation of the prosociality rates by cases across 6 blocks of trials in the observer group. 

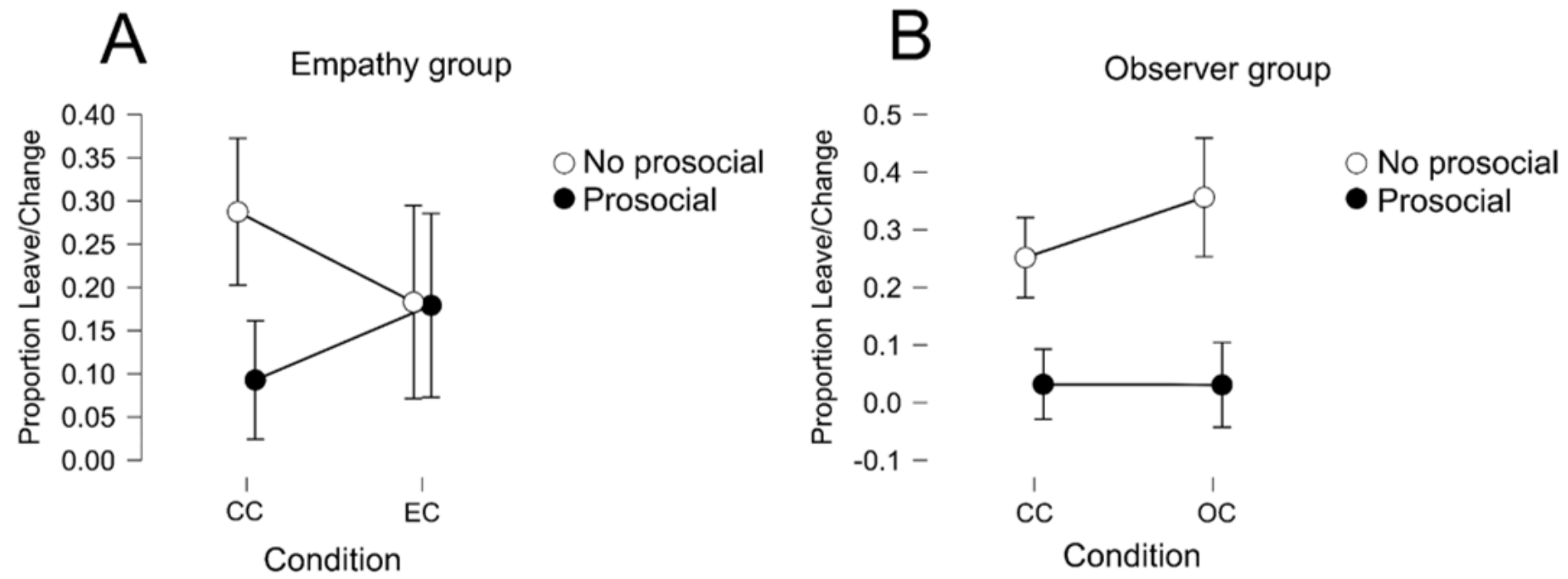

Figure 4

(A) Proportion in which participants of the Empathy group confirm or leave the opposite choice. (B) Proportion in which participants of the observer group confirm or leave the opposite choice
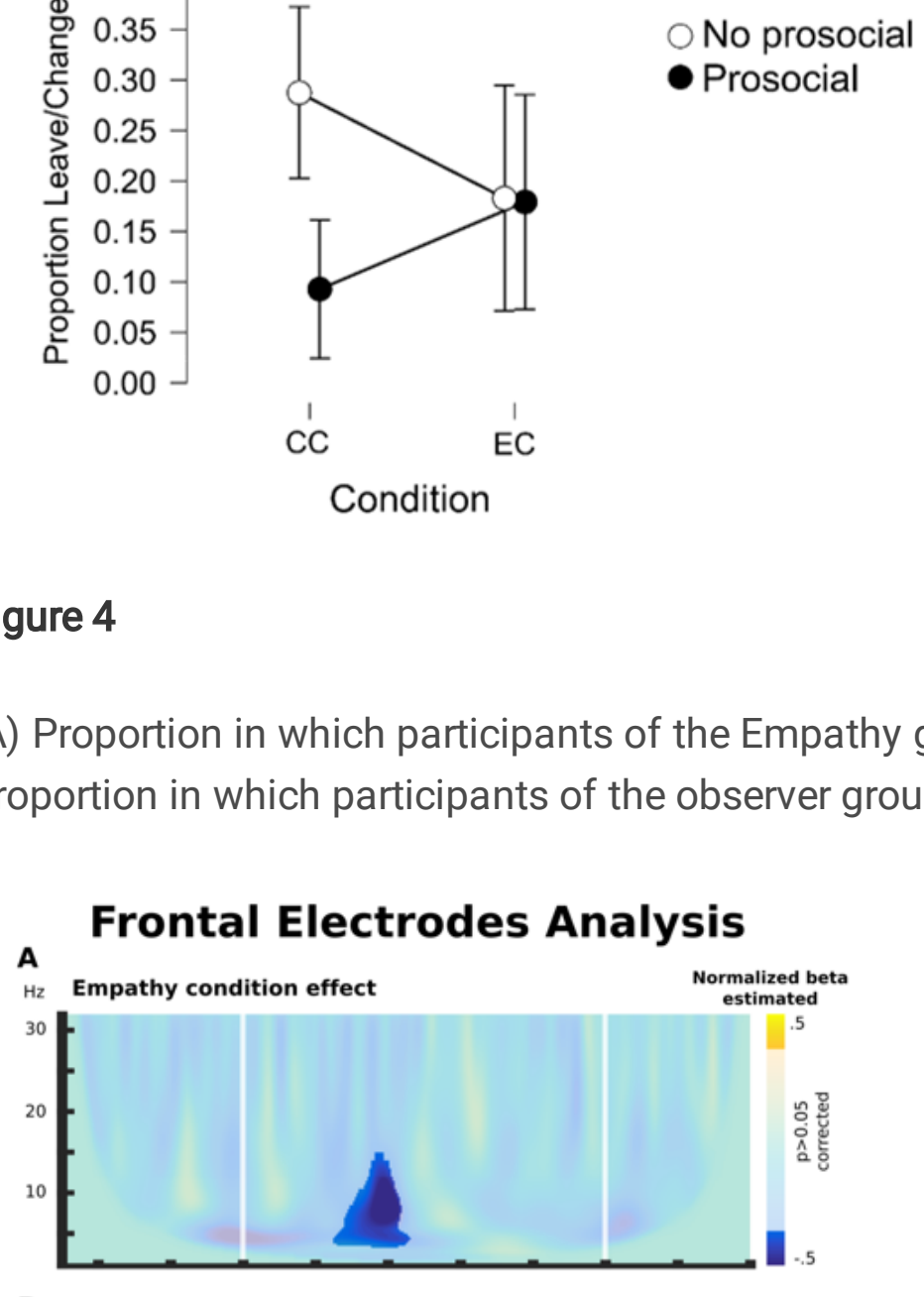
(A) Difference between the control and empathy conditions in the correlation between oscillatory brain activity in the frontocentral electrode (white rectangle in $\mathrm{E}$ ) and the unequal distribution to the other player during the decision time. (B) Difference between the control and observer conditions in the correlation between the oscillatory brain activity in the frontocentral electrode (white rectangle in $\mathrm{E}$ ) and the unequal distribution to the other player during the decision time. (C) Between-group comparison of the difference between the control and experimental conditions in the correlation between the oscillatory brain activity in the frontocentral electrode (white rectangle in $\mathrm{E}$ ) and the unequal distribution to the other player during the decision time. (D) Significant cluster emerging by means of a without a priori whole scalp analysis for the contrast shown in C. (E) Scalp distribution of the theta modulation in the time-frequency windows highlighted in $\mathrm{C}$ and its source estimation. ( $\mathrm{F}$ ) Another significant cluster emerging by means of a without a priori whole scalp analysis for the contrast shown in C. (G) Scalp distribution of the theta modulation of the cluster shown in $\mathrm{F}$ and its source estimation. 